\title{
A Carrier Signal Approach for Intermittent Fault Detection and Health Monitoring for Electronics Interconnections System
}

\author{
Syed Wakil Ahmad \\ EPSRC Centre for Innovative Manufacturing in Through- \\ life Engineering Services, Cranfield University, MK 43 \\ OAL, UK \\ Dr. Suresh Perinpanayagam \\ Integrated Vehicle Health Management Centre, Cranfield \\ University, MK43 0QF,UK
}

\author{
Prof. Ian Jennions \\ Integrated Vehicle Health Management Centre, Cranfield \\ University, MK43 0QF, UK \\ Dr. Mohammad Samie \\ Integrated Vehicle Health Management Centre, Cranfield \\ University, MK43 0QF, UK
}

\begin{abstract}
Intermittent faults are completely missed out by traditional monitoring and detection techniques due to nonstationary nature of signals. These are the incipient events of a precursor of permanent faults to come. Intermittent faults in electrical interconnection are short duration transients which could be detected by some specific techniques but these do not provide enough information to understand the root cause of it. Due to random and non-predictable nature, the intermittent faults are the most frustrating, elusive, and expensive faults to detect in interconnection system. The novel approach of the author injects a fixed frequency sinusoidal signal into electronics interconnection system that modulates intermittent fault if persist. Intermittent faults and other channel effects are computed from received signal by demodulation and spectrum analysis. This paper describes technology for intermittent fault detection, and classification of intermittent fault, and channel characterization. The paper also reports the functionally tests of computational system of the proposed methods. This algorithm has been tested using experimental setup. It generate an intermittent signal by external vibration stress on connector and intermittency is detected by acquiring and processing propagating signal. The results demonstrate to detect and classify intermittent interconnection and noise variations due to intermittency. Monitoring the channel in-situ with low amplitude, and narrow band signal over electronics interconnection between a transmitter and a receiver provides the most effective tool for continuously watching the wire system for the random, unpredictable intermittent faults, the precursor of failure.
\end{abstract}

Keywords-NFF; Intermittent; Intermittency; Fault detection; Health Monitoring

\section{INTRODUCTION}

An intermittent fault (IF) is an electrical spike that develops from ageing of electric interconnects, cuts, rubs, or loose contacts, and manifests itself intermittently in an unpredictable manner. If these are not detected on time or at the early stage, it would gradually lead to permanent fault and are also safety critical [1]. This also lead to, many other problems for example delayed or cancellation of flights, electrical arc or spark that could lead disaster that progressed from IFs.

Manufacturing imperfections, poor design and system degradations are main causes of intermittent faults [2] Although Sheng et al are disagree that intermittent faults are precursor of permanent failure [3] but S. Bryan et al says that intermittent faults are precursor of hard failure [4]. These both statements could be true, depends upon causes of intermittence. IF due to system/component degradation are precursor of permanent faults but marginal design or manufacturing imperfection are not signs of hard failure. Irrespective of causes; IFs are random and non-reproducible incidents, and are most frustrating, elusive, and expensive faults to detect and locate in wiring / interconnection systems.

IFs are identified by visual or traditional instruments for electronic/electrical interconnects. It has also been reported that conventional test equipment, which is required to carry out the fault investigation, are not always successful. This can be due to the fact that the necessary levels of confidence and efficiency are inappropriate in the many industries which are suffering No Fault Found (NFF) failures [5]. If testability as a design characteristic was successful, perhaps NFF would not be so problematic. This is particularly evident in the case of attempting to detect and isolate intermittent faults at a test station the ability to test for short duration non-stationary intermittency at the very moment that it re-occurs using conventional methods is so remote that it will almost certainly result in a NFF. The one major issue with designing component testability is that the focus is on functionality and integrity of the system.

There are many test equipment that are used to detected anomalies in electrical interconnection systems. The more common ones include multi-meters that detect steady or invariant signals. On the other hand, digital oscilloscopes, and spectrum analyzers are used to monitor time domain and frequency domain time invariant signals. Problem with an intermittent fault is that it occurs for only a short duration and it is time variant, making it difficult to detect unless a very 
high sample rate it used. This goes beyond the capabilities of typical test equipment. The current state-of the-art in intermittent fault detection during maintenance testing includes latching continuity testing, analogue neural network technology and time domain reflectometry.

There are various disadvantages of these techniques: to halt operation for inspection, hard to capture or watch on oscilloscope or voltmeter as well as ineffectiveness due to many inspection points and some time being in the location frequently hard to reach or observe. These are unable to detect the fault in many cases since the duration of the fault was often short and not consistent. System would behave normally and it would find the interconnection/wire system normal or NFF status. Therefore, it is easy for the observer or instrument to miss the occurrence of intermittent fault.

Much research has been done on reflectometry wiring fault detection and that is used for high power electrical wirings and could not be used for interfaces and lose solder joints or for other electronics circuits. The concept of reflectometry relies on transmitting electromagnetic waves across the wire and observe the reflections. These reflections depends upon the variation of impedance in the wire system as $\frac{Z_{1}-Z_{2}}{Z_{1}+Z_{2}}$, where $Z_{1}$ and $Z_{2}$ are impedances of two electrical mediums [6] Time between the incident and the reflected wave is used to locate the fault. Magnitude of reflections are used to determine if it is a potential fault or not. These techniques have drawbacks for modern electronics / electrical system that any change in the wire material (e.g., connection in circuit) reflects the incident waves resulting in incorrect fault determination. These techniques usually requires high voltage pulses.

Recently, direct-sequence spread-spectrum (DS-SS) signals are used instead of high voltage signals employing digital signal processing techniques to find and locate electrical faults [7]. Taylor and Faulkner proposed directsequence spread spectrum modulation on power line carrier, and outlined optimal signal processing techniques and frequency domain correlation techniques for the on-line test in high voltage line [8]. Lately, slightly different use of spread spectrum was reported from the research result of on detecting live wire problems [9]. These techniques work on reflectometry, and it solves the need to use low voltage signal, that does not interfere with online signals and could be used in-situ, but still there is a problem of reflection occurring at all points of interconnections in the circuit. So this technique is inadequate for interconnecting system, where there are many interfaces and connectors. This is also not suitable to use for electronic circuits i.e. for PCBs, solder joints, interfaces, and similar interconnecting systems. Otherwise, the injected signal would be reflected from both ends and result in a combined, distorted, and reflected false signal due to impedance mismatch.

The novel approach of IF detecting and characterization has been developed by the author to overcome above mentioned issues and it is very different from traditional diagnostic methods. Novelty of the proposed new technique is the fact that signs of IF intrinsically modulated on a carrier signal, in compare with healthy wired communication channel and interconnection system. In healthy communication link carrier signal propagates without any changes that affect amplitude/phase/frequency of signal but with Additive White Gaussian Noise (AWGN). The proposed technique aims to look at signature of intermittency as a modulated message on carrier, and employ demodulation techniques to explore behavior of aged channel/interconnections.

The new approaches of the author send a sinusoidal carrier to interconnecting system and demodulate the received signal from interconnection channel for IF detection and feature extraction to find the root cause of problem. This could be used for multipoint of electrical/ electronic interconnection system and diagnose the health status of the wire after dedemodulation to retrieve an intermittent signature of channel. The essence of this approach is using communication modulation techniques to detect and electrical interconnection system. The transient caused by the intermittent fault in the wire would disrupt the signal sent over interconnection from a transmitter, and thus arriving signal at the receiver would contain intermittent signal information. When intermittent signals are found it will extract IF information by demodulation algorithms. The features of amplitude, phase, and frequency are computed by AM (Amplitude Modulation), PM (Phase Modulation) and FM (Frequency Modulation) demodulation schemes. The benefits of computing phase, amplitude, and frequency of IF could be used to classify intermittent signal for root cause analysis, and degradation monitor.

In the next section, we describe the communication technology and its devised method for detection and computation of fault's information in terms of duration, occurrence frequency, and channel noise. Then, third section describes, devised communication approach for IF detection using demodulation computations. Fourth section describes the test rig and application. Following section describes the results and validations of algorithm then last section concludes this paper.

\section{COMMUNICATION APPROACH FOR INTERMITTENT FAULT DETECTION}

Related to fault detection, author has used radar communication approach where it sends blank carrier signal and extras desire information from received signal. Intermittent characteristics of channel will change the propagating signal and these intermittent signature could be computed by removing original signal. Carrier modulation / demodulation concept is being used to as sounding techniques to extract IF signature.

There are many carrier modulation schemes but fundamentally there are three modulations schemes called amplitude modulation (AM), frequency modulation (FM) and phase modulation (PM). In AM, the amplitude of carrier signal changes according to input signal and this concept is being used that if there is an intermittent open/close it changes the amplitude of carrier signal. Similarly phase and frequency changes could be computed by using PM and FM demodulation concept. 


\section{A. Theory and formulation}

Any AM, PM or FM signal $x(t)$ can be written as shown in equation 1

$$
x(t)=R(t) \cos (\omega t+\varphi(t))
$$

In equation (1) $\mathrm{R}(\mathrm{t})$ is the envelope of signal (amplitude of signal as function of time), $\omega$ is angular frequency, and $\varphi(t)$ is a phase of signal at t time.

For $\mathrm{AM} \varphi(t)$, and $\omega$ are constant only envelope $R(t)$ is time variant, thus equation (1) can be written as below

$$
x(t)=(C+m(t)) \cos (\omega t) . .(2)
$$

In equation (2) $R(t)$ envelope is replaced to $C+m(t)$ where $m(t)$ is amplitude of base signal, in our case this is an IF signal, and " $C$ " is carrier amplitude.

The IF signal $m(t)$ could be extracted by simple diode rectification and low or band pass filtration for analogue circuits and could be compute digital filtering / modulation algorithms. Filter band must be according to the band range of IF signal else information of IF will be lost.

For PM and FM the amplitude envelope will remain constant but it varies the phase/frequency. For FM/PM demodulation, the signal is fed into a Phase Loop Lock (PLL) and the error signal is used as the demodulated signal.

\section{NOVEl Fault Dection Algorithm}

In wireless communication, to model channel behavior they measure its propertied by sending and receiving wireless signal, are called channel sounding techniques [10]. Author

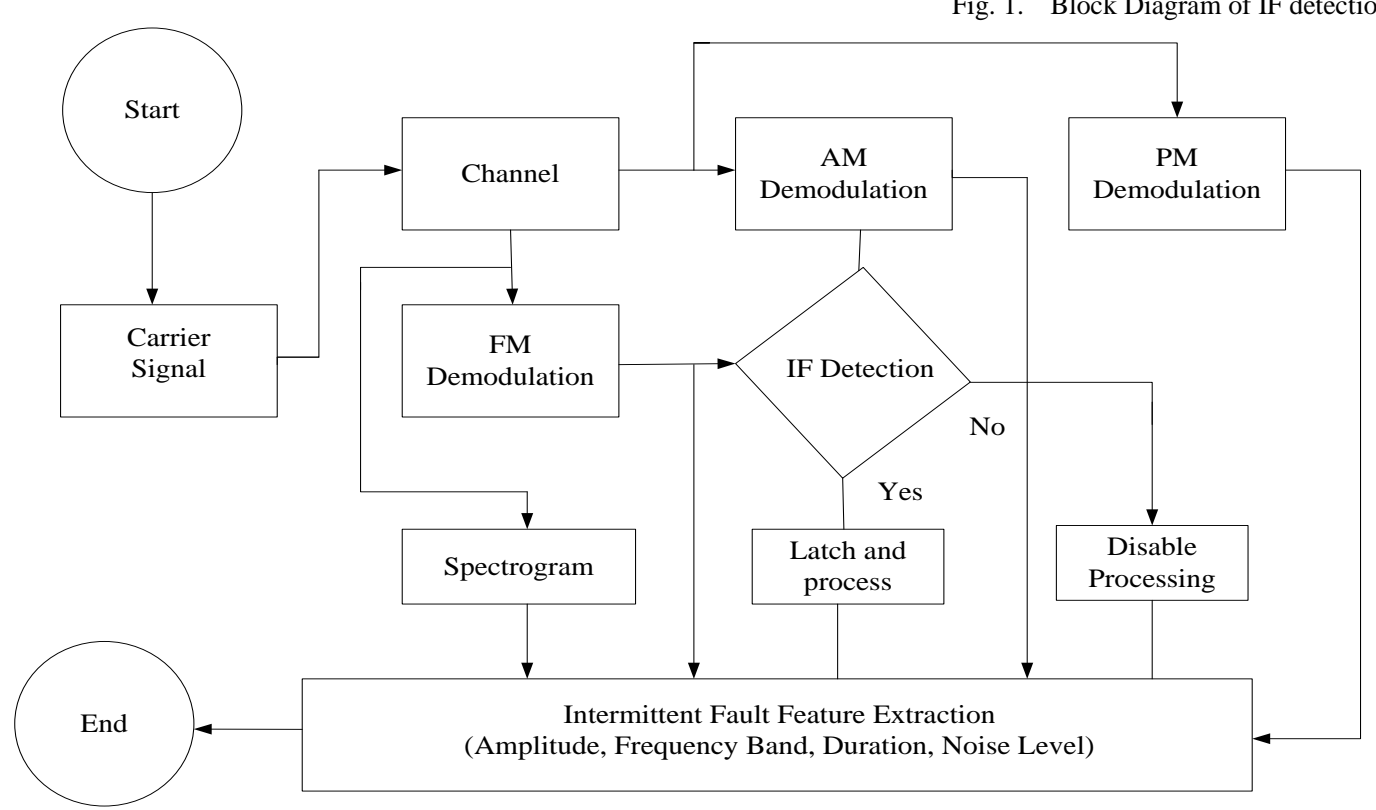

Fig. 2. Intermittent Fault Detection Algorithm

Figure 2 shows the flow diagram of this algorithm. It starts with suitable selected carrier signal that fulfil the required resolution; $1 \mathrm{k} \mathrm{Hz}$ sine wave is selected to give one millisecond resolution that is suitable for our repetitively producing IFs test rig. The advantage of using one millimetre resolution, is that it will eliminate debouching harmonics but has adapter similar method to measure an intermittency in electric/electronics interconnection systems. To measure IF and its properties it sends and receives suitable signal through interconnection system. A novel algorithm has been developed to compute intermittency for IF detection and classification. Its features of amplitude, frequency, and phase are computed using AM, PM, and FM demodulation algorithms while Fast Fourier Transform (FFT) computes its spectrum. This algorithm has been shown in Fig. 1, it consists of signal source (carrier frequency), intermittent channel (test rig), demodulating unit, digital filter, IF detection using AM, FM and PM algorithms. It counts an intermittency and IF fault detection turn on. Each fault duration and frequency of occurrence are stored in output buffer.

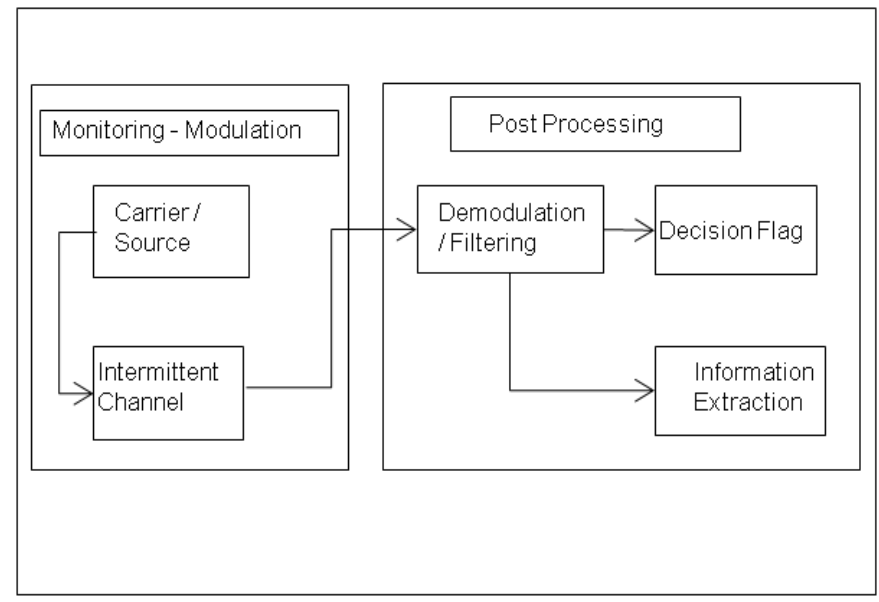

Fig. 1. Block Diagram of IF detection Algorithm

if high resolution is required for less frequent IF, carrier frequency could be increased accordingly i.e. resolution is inversely proportional to carrier frequency. Carrier signal propagates through interconnection system to terminating point to complete a circuit. IF detection unit constantly process carrier signal to compute IF and dynamics. Processing 
unit demodulates using amplitude, frequency, and phase demodulation schemes. The spectrogram is also computed to check the bandwidth and noise level. Frequency, amplitude and bandwidth information are used to detect IF.

To make IF detection decision AM and FM demodulation techniques are used, if there is not any IF then it will disable the feature extraction and memory but if IF is detected it latches the signal and extracts its feature.

This also save computation power and memory. It also computes the amplitude, bandwidth, noise level, and time information of signal when decision flag is on.

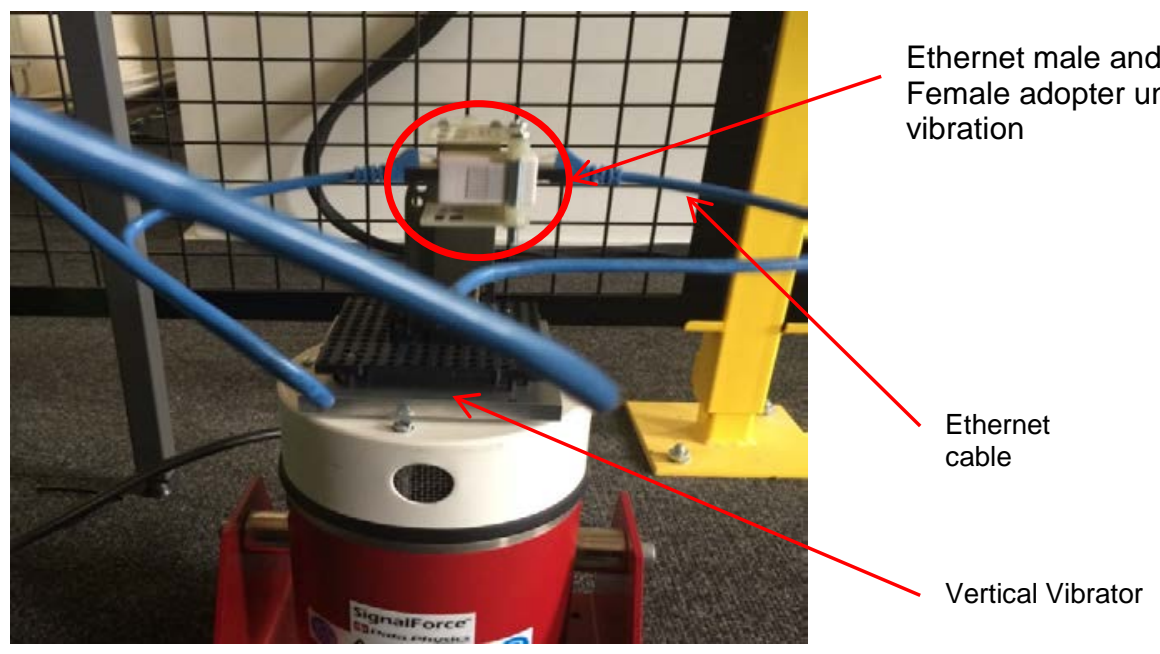

Fig. 3. Ethernet Male and Female Socket with Cable Connection as an Intermittent Test Rig

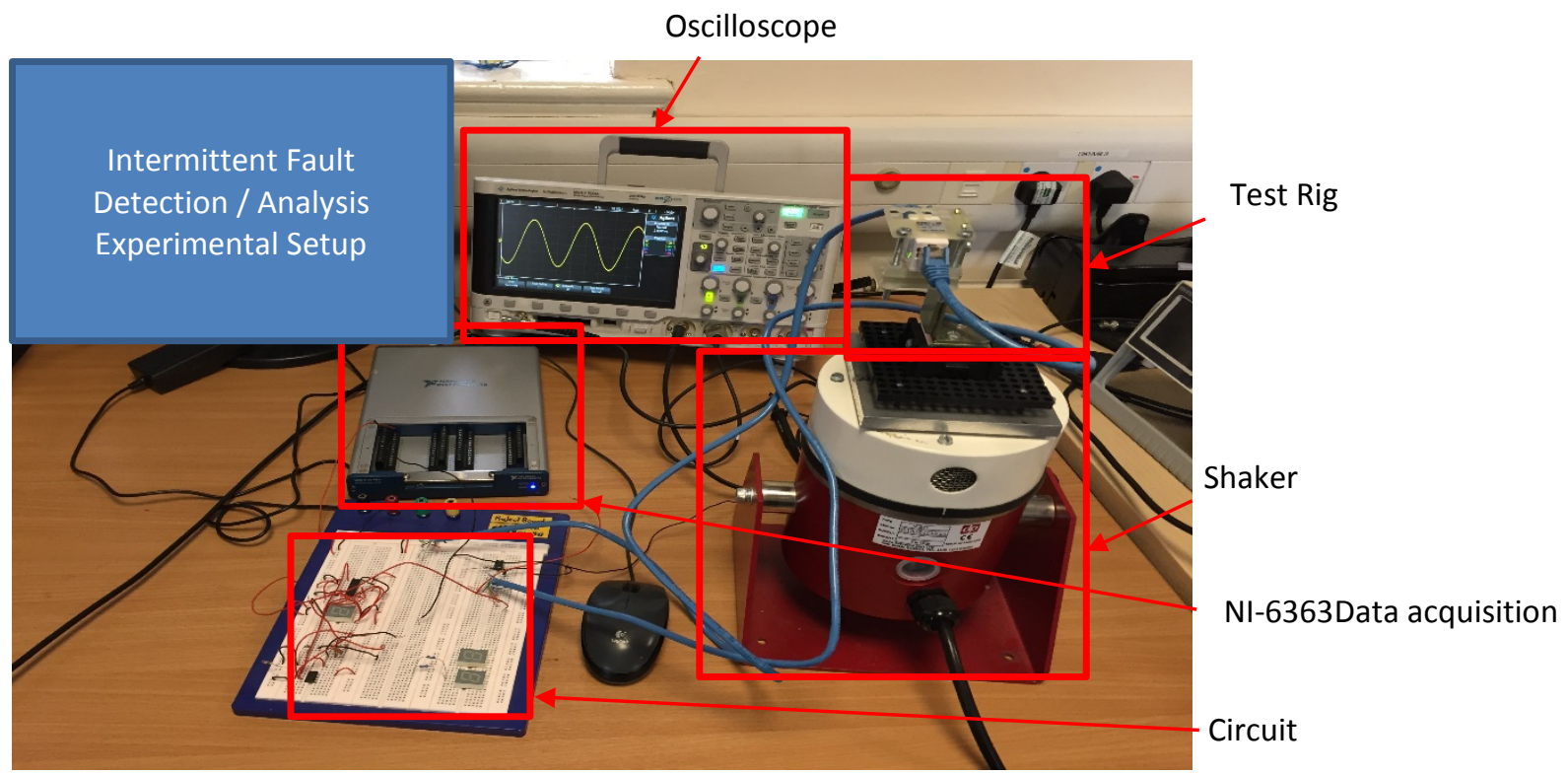

Fig. 4. Experimental Setup for Intermittent Fault Detection

\section{APPLICATION \& CASE STUDY}

RJ45 Ethernet socket with Ethernet cable/plug under external vibration is used to generate intermittence in the connection. A Female RJ45 Ethernet socket is used to hold it with assembly on shaker that Connector can vibrate as shown in Fig. 3. The grid has been installed on the shaker by screws and a metal plate as shown Fig. 3.This Ethernet connection assembly is used to produce the intermittent fault under vibration. Other ends of Ethernet cable are connected to a circuit and data acquisition system. A complete circuit setup is shown in Fig. 4. It consists of a test rig, oscilloscope, and data acquisition system.

Ethernet male and

Female adopter under vibration

Ethernet

Vertical Vibrator 
This oscilloscope has four channels, $4 \mathrm{G}$ bits/second sample rate, $200 \mathrm{MHz}$ bandwidth and built-in function generator that can output variety of signals but we used $1.00 \mathrm{~K}$ $\mathrm{Hz} 3 \mathrm{v}$ peak to peak sinusoidal signal as voltage source to voltage divider circuit. The NI-6363 data acquisition card can acquire up to 2 mega samples per second.

The input sine wave of one kilo hertz is propagates through test rig to receiver. To detect an IF and other information, the data is acquired using NI data acquisition card. Received data is being processed using FFT, AM, FM and PM demodulation algorithms. The decision has been taken if there is an IF fault or not; if there is an IF then its noise level, duration and frequency is calculated for IF classification or analysis.

\section{SIMULATION AND VALIDATION}

The algorithm has been validated by acquiring data from above mention experimental setup and processed in matlab using algorithm described in section 2.

Input carrier signal at $1 \mathrm{k} \mathrm{Hz}$ frequency, to electronic interconnection system is shown in 5 . This propagated through a test rig under vibration as shown in Fig. 4

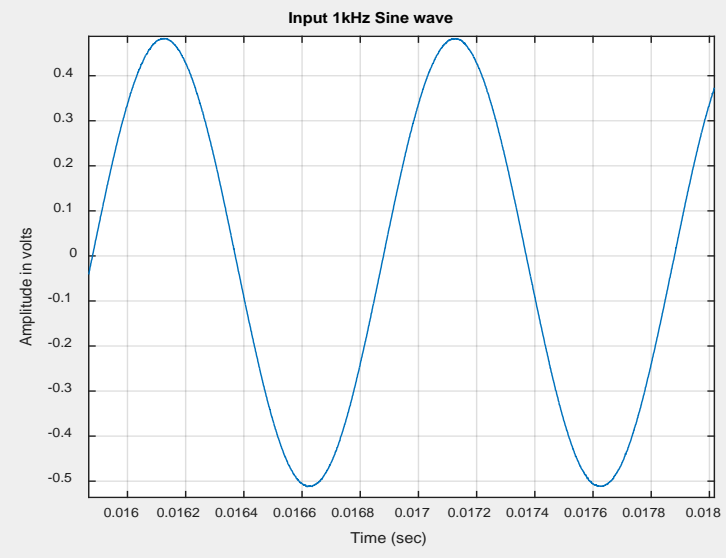

Fig. 5. Input sine wave to unit under test

Shaking test rig adds an intermittency and other noises to a carrier signal due to lose electrical / electronic circuit. Received signal is shown in Fig. 6. This shows that how IF effect on propagating signal. This is output of channel as described in Figure 4.

To detect IF and to extract its feature, it has been demodulated with respect to amplitude, frequency and phase. Amplitude demodulation gives information where amplitude of signal drops due to intermittent discontinuity while change in frequency can be calculated using frequency demodulation. Intermittent fault also changes the phase of signal due to nonlinear discontinuities and could be calculated using phase demodulation.

Fig. 7 shows AM demodulated signal that gives an intermittent signal with twenty spikes of an intermittent fault of a connection shaking at $20 \mathrm{~Hz}$. The amplitude of these spikes shows the change in the amplitude with respect to carrier signal at that instant.

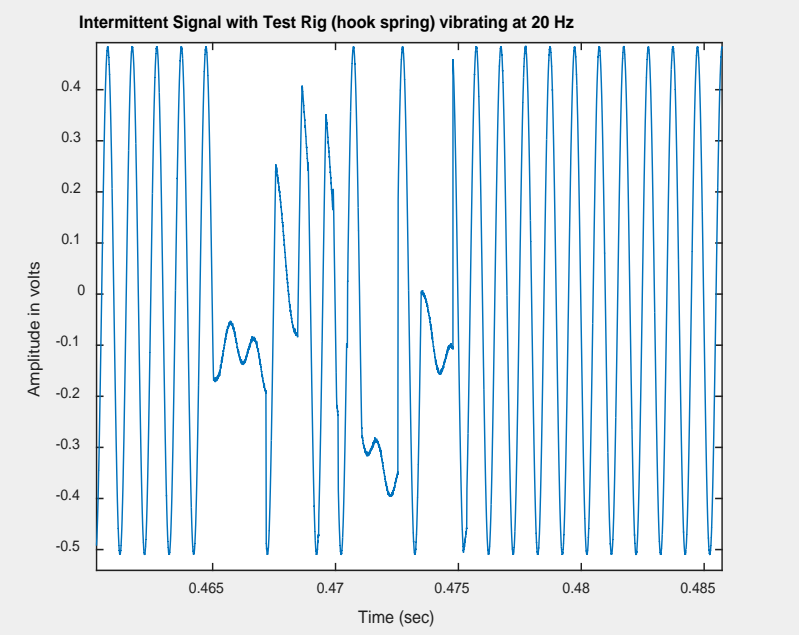

Fig. 6. Received noisy signal with IF information

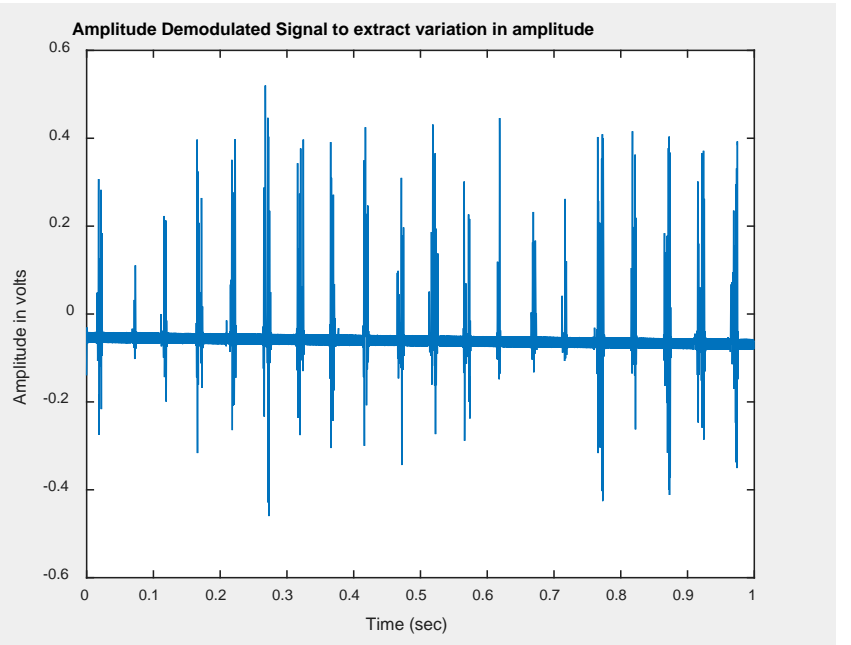

Fig. 7. AM Demodulated Signal

Fig. 8 shows frequency changes with respect to carrier signal. The magnitude indicates changes in the frequency at that instant. The feature of change in frequency are used to calculate the duration of an intermittent interval by subtracting it from carrier frequency and taking inverse. The IF detection decisions are made by comparing both $\mathrm{AM}$ and FM demodulated signals and these are also used to calculate its duration and frequency of intermittent fault. It only enable processing unit then there is an intermittent interval as described in Figure 2. The phase change is calculated by phase demodulation as shown in Fig. 9. It gives an information that how phase of intermittent signal has changed. This could be used to study that how an IF effect the signal and change the phase of transmission and adds noise to signal.

The power spectrum of IF signal is shown in Fig. 10. The carrier frequency and intermittent signal are shown in this figure at different frequencies. The normalized frequency has peaks at 0.05 and 0.001 ; these corresponds $1000 \mathrm{~Hz}$ and $20 \mathrm{~Hz}$ frequencies when samples at 20k sample/second sampling frequencies. This power spectrum shows the power spectrum of its signals at carrier and around $20 \mathrm{~Hz}$ intermittent signal's spectrum. 


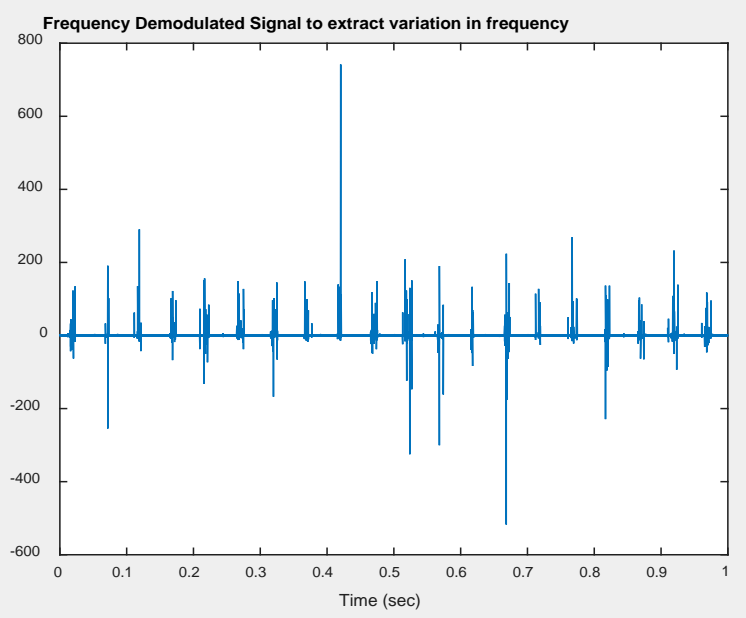

Fig. 8. Frequency Demodulated Signal with $20 \mathrm{~Hz}$ Shaking Connector

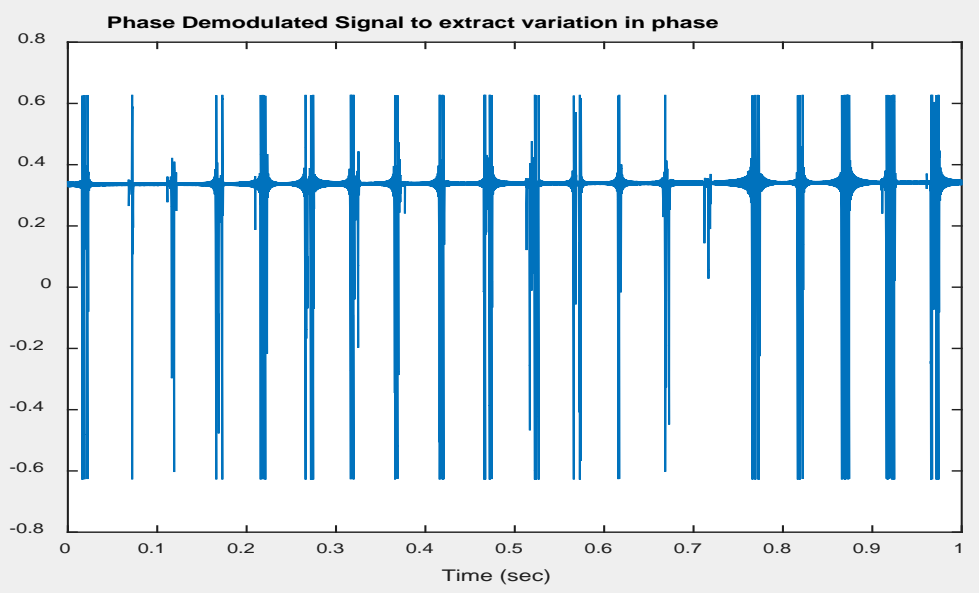

Fig. 9. Phase Demodulated Signal with shaking $20 \mathrm{~Hz}$ External Vibration to a connector

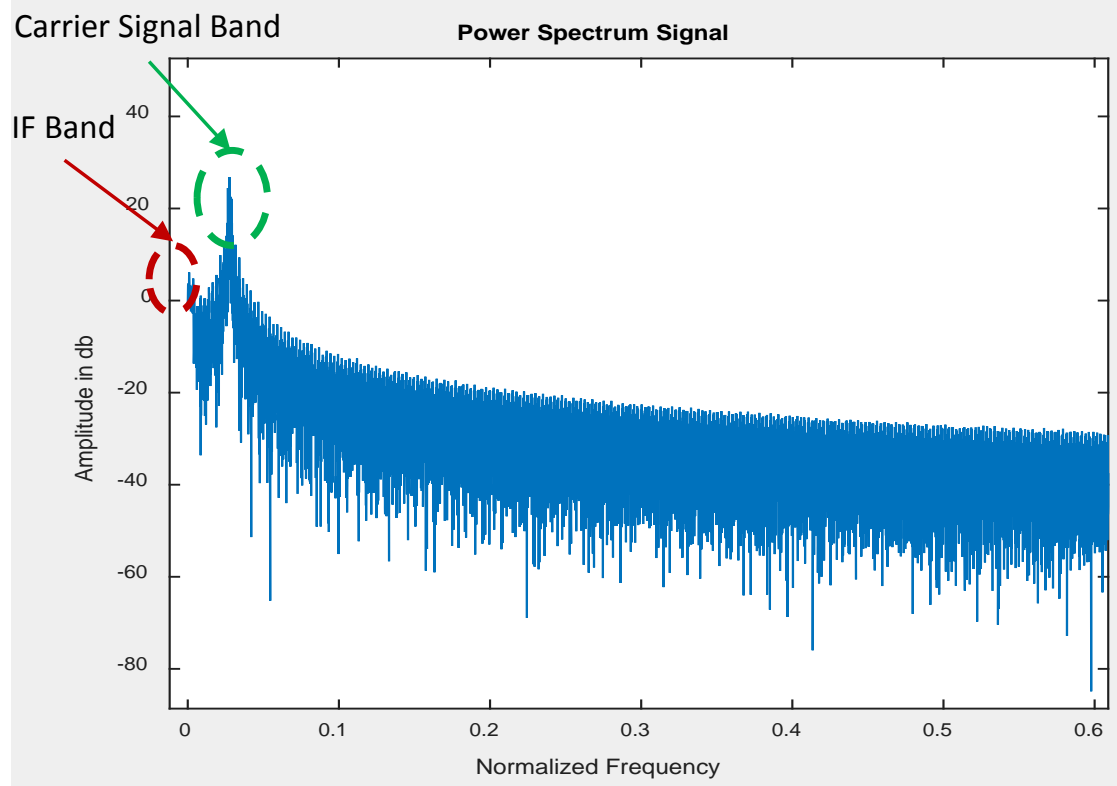

Fig. 10. Spectrum of Intermittent signal 
The algorithm described in section 3 has been verified and it gives IF detection and its feature. In this experiment IF correlates with external vibration as we have seen that at 20 hertz shaking lose connection gives us 20 hertz intermittency but the duration and magnitude of IF are not identical for all faults.

\section{CONCLUSION}

NFF can be overcome by using in-situ health monitoring algorithm to check it for abnormalities in the interconnection system. An intermittent signal can be detected and classified by using classical demodulation schemes. Amplitude, frequency, and phase variation has been extracted by AM, FM and PM modulation schemes, which gives intermittent channel information.

We have seen that amplitude and frequency variations are very useful for root cause analysis. It also highlights that phase information are not very helpful to understand the exact cause. This algorithm also gives IF signal's characteristics of amplitude, and frequency variation that could help to understand its effects on the system performance. Power spectrum is a very useful tool and that could be used to determine the frequency of intermittency as in experiment IF could be seen at $20 \mathrm{~Hz}$ because test rig was shaken at this frequency. Although it is not necessary that spectrum exactly relates to external vibration but could be used to understand the root cause; if it is due to vibration or due to other noise.
Further work could be carried out using filter banks to segment different possible bands of spectrum and this could be used to detect intermittence, and to find root cause.

\section{REFERENCES}

[1] A. Correcher, E. García, F. Morant, E. Quiles and L. Rodríguez, "Intermittent Failure Dynamics Characterization," IEEE TRANSACTIONS ON RELIABILITY, vol. 61, no. 3, pp. 649-658, 2012.

[2] W. Syed, S. Khan and P. Philips, "Intermittent fault finding strategies," in CIRP, 2013.

[3] S. Sheng, X. Mingqing, L. Zhao and R. Tingting, "Misunderstandings on intermittent failures and LCP-based description of intermittent failures," Zhangiiaijie, August 2014.

[4] S. Bryan, B. Floyd, O. Nathan and S. Brent, "Intermittent Fault Detection and Isolation System," Sait Lake City, 2008.

[5] H. Qi, S. Ganesan and M. Pecht, "No-Fault Found and Intermittent Failures in Electronic Products," Microelectronics Reliability Elsevier, vol. 48, no. 5, pp. 663-674, 2008.

[6] S. Paul, F. Cynthia and et-al, "Spread Spectrum Sensors for Critical Fault Location on Live Wires," IEEE Sensors Journal, June 2005.

[7] P. Smith, F. Cynthia and J. Gunther, "Analysis of Spread Spectrum Time Domain Reflectometry for Wire Fault Location," IEEE SENSORS JOURNAL, vol. 5, no. 6, pp. 1469-1478, December 2005.

[8] C. Kim, "Detection and Location of Intermittent Faults by Monitoring Carrier Signal Channel Behaviour of Electrical Interconnection System," in Electric Ship Technologies Symposium, 2009. ESTS 2009. IEEE, Baltimore, MD, 20-22 April 2009.

[9] F. Cynthia, S. Paul, L. Chet and et-al, "Spread Spectrum for Critical Fault Location on Live WireNetworks," Structural Control Health Monitoring,Wiley InterScience, pp. 257-267, 2005.

[10] A. F. Molisch, "Andreas F. Molisch," in Wireless Communications, 2nd Edition, Willey, 2012, p. 884. 